\title{
Measuring time-domain spectral induced polarization in the on-time: decreasing acquisition time and increasing signal-to-noise ratio
}

\author{
Per-Ivar Olsson ${ }^{\mathrm{a}, *}$, Torleif Dahlin ${ }^{\mathrm{a}}$, Gianluca Fiandaca ${ }^{\mathrm{b}}$, Esben Auken ${ }^{\mathrm{b}}$ \\ a Engineering Geology, Lund University, Sweden \\ b Department of Geosciences, Aarhus University, Denmark
}

\section{A R T I C L E I N F O}

\section{Article history:}

Received 16 April 2015

Received in revised form 28 July 2015

Accepted 26 August 2015

Available online 2 September 2015

\section{Keywords:}

Spectral

Induced polarization

Signal-to-noise ratio

SNR

Waveform

Duty cycle

Cole-Cole

\begin{abstract}
A B S T R A C T
Combined resistivity and time-domain direct current induced polarization (DCIP) measurements are traditionally carried out with a 50\% duty cycle current waveform, taking the resistivity measurements during the on-time and the IP measurements during the off-time. One drawback with this method is that only half of the acquisition time is available for resistivity and IP measurements, respectively. In this paper, this limitation is solved by using a current injection with $100 \%$ duty cycle and also taking the IP measurements in the on-time. With numerical modelling of current waveforms with $50 \%$ and $100 \%$ duty cycles we show that the waveforms have comparable sensitivity for the spectral Cole-Cole parameters and that signal level is increased up to a factor of 2 if the 100\% duty cycle waveform is used. The inversion of field data acquired with both waveforms confirms the modelling results and shows that it is possible to retrieve similar inversion models with either of the waveforms when inverting for the spectral Cole-Cole parameters with the waveform of the injected current included in the forward computations. Consequently, our results show that on-time measurements of IP can reduce the acquisition time by up to $50 \%$ and increase the signal-to-noise ratio by up to $100 \%$ almost without information loss. Our findings can contribute and have a large impact for DCIP surveys in general and especially for surveys where time and reliable data quality are important factors. Specifically, the findings are of value for DCIP surveys conducted in urban areas where anthropogenic noise is an issue and the heterogeneous subsurface demands timeconsuming 3D acquisitions.
\end{abstract}

(c) 2015 Elsevier B.V. All rights reserved.

\section{Introduction}

Combined direct current (DC) resistivity and time-domain (TD) induced polarization (IP) measurements are traditionally carried out using a 50\% duty cycle waveform for current injection, taking the resistivity measurements during the on-time and the IP measurements during the off-time. Recently, however, developments in time-domain hardware have made it possible to perform detailed monitoring and analysis of the measuring procedure of DCIP acquisition. As a result of this development, it has been suggested that a $100 \%$ duty cycle waveform could be used in time-domain IP, with the IP (spectral) information retrieved from the on-time (Dahlin and Leroux 2010). Furthermore, in frequency-domain, a $100 \%$ duty cycle waveform has been successfully used for measuring IP, although retrieving limited spectral information (Zonge et al. 2005, Zonge et al. 1972).

Alongside the hardware improvement, a corresponding development on the software side has taken place. Inversion algorithms that use the current waveform and the full IP decays for extracting the

\footnotetext{
* Corresponding author at: Teknisk Geologi, Lunds Tekniska Högskola, P.O. Box 118, SE22100 Lund, Sweden.

E-mail address: per-ivar.olsson@tg.lth.se (P.-I. Olsson).
}

spectral IP content from TD data have been proposed (Fiandaca et al. 2012, 2013), with applications for landfill characterization (Gazoty et al. 2013, Gazoty et al. 2012a, Gazoty et al. 2012b) and for mapping CO2 transport (Doetsch et al. 2015a) and permafrost seasonal variations (Doetsch et al. 2015b).

For these applications, as well as for others, the use of a 100\% duty cycle waveform for DCIP measurements will have substantial advantages compared with the traditionally used 50\% duty cycle regarding acquisition time efficiency and signal level. These benefits are highly relevant for the applied DCIP surveys conducted by commercial companies where time and reliable data quality are important factors. In addition, DCIP surveys conducted in urban areas where anthropogenic noise sources are present and the heterogeneous subsurface demands timeconsuming 3D acquisitions will have extra benefit from the $100 \%$ duty cycle waveform and on-time measurements of IP. To date, it has not been shown whether the $100 \%$ duty cycle waveform can be successfully used in TDIP measurements, nor if spectral information can be retrieved in the inversion.

This paper demonstrates that the use of a $100 \%$ duty cycle waveform and on-time measurements of the spectral IP information is possible for both synthetic and field DCIP cases. The aforementioned hardware and software developments are used for comparing 50\% and 100\% duty 
cycle waveforms, both in terms of IP spectral information and signal to noise ratio. Section 2 introduces the $100 \%$ duty cycle waveform in details, followed by sections presenting results and discussion on the synthetic modelling and a field comparison. The paper ends with a brief conclusion.

\section{100\% duty cycle}

A step-response for an exemplary homogenous half-space Cole-Cole model was generated with the spectral forward response and inversion code Aarhusinv (Auken et al. 2014) and used for creating synthetic fullwaveform data of the $50 \%$ and $100 \%$ duty cycle waveforms (Fig. 1 ) by the principle of superposition (Fiandaca et al. 2012).

It can be noted that for the $50 \%$ duty cycle waveform with IP measurements during the off-time, the measured IP response decays with time, while for the $100 \%$ duty cycle waveform with IP measurements during the on-time, the potential increases during the acquisition. For an easier comparison between the $50 \%$ and $100 \%$ signals, this paper uses the $V_{D C(j)}$ value (retrieved from a time period at the end of each pulse $j$ ) for defining the $100 \%$ IP decay for pulse $j, V_{I P 100 \%(j)}$, as the difference between the $V_{D C(j)}$ value and the measured IP response $V_{\text {response }(j)}$ (Eq. (1)):

$V_{\mathrm{IP} 100 \%(j)}=V_{\mathrm{DC}(j)}-V_{\text {response }(j)}$.

The individual $100 \%$ IP decays derived with Eq. (1), $V_{I P 100 \%(j)}$, are averaged according to Eq. (2), making use of the negative or positive sign of the decays:

$V_{\mathrm{IP} 100 \%, \text { average }}=\frac{1}{n_{\text {pulses }}} \sum_{j=1}^{n_{\text {pulses }}}(-1)^{j+1} \cdot V_{\mathrm{IP} 100 \%(j)}$.

For the 50\% duty cycle, a normalization of the IP response is traditionally achieved by dividing the IP response potential with the VDC value (VIP/VDC) and presenting the IP response in $\mathrm{mV} / \mathrm{V}$ (note that this normalization does not define the "normalized chargeability" described for instance by Slater and Lesmes (2002), but is a common unit change). A corresponding normalization for the $100 \%$ duty cycle is also suggested in this paper through Eq. (3):

$V_{\mathrm{IP} 100 \%, \text { normalized }}=\frac{V_{\mathrm{IP} 100 \%, \text { average }} \cdot n_{\text {pulses }}}{V_{\text {DC,average }} \cdot\left(2 \cdot n_{\text {pulses }}-1\right)}$

where $V_{D C \text {, average }}$ represents the $\mathrm{DC}$ value averaged over all the pulses in analogy to Eq. (2). The normalization of Eq. (3) differs from the standard IP normalization by the presence of the $\frac{n_{\text {pulses }}}{\left(2 \cdot n_{\text {pulses }}-1\right)}$ factor, which has been introduced in order to facilitate the numerical comparison of $50 \%$ and $100 \%$ IP duty cycles normalized data.

In fact, as can be seen in Fig. 2A, the magnitude of the chargeability for the $100 \%$ duty cycle waveform is initially almost twice the magnitude of the $50 \%$ duty cycle waveform if compared without normalization (presented in volt). This doubling is due to the superposition of the off-time IP decay of the $50 \%$ duty cycle waveform and the corresponding on-time IP build-up. With the $100 \%$ duty cycle waveform, these two responses are superimposed on each other and measured as one. Another way to look at this is to consider that the change in current causing the IP response is doubled when using the 100\% duty cycle waveform instead of the $50 \%$ waveform for all the pulses except the first, which is initiated from the same current level (i.e. zero ampere,

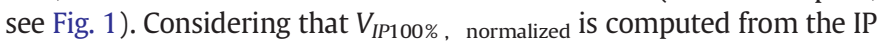
responses averaged over all the pulses, the $\frac{n_{\text {pulses }}}{\left(2 \cdot n_{\text {pulses }}-1\right)}$ factor in Eq. (3) standardizes the $100 \%$ duty cycle response to the current changes of the $50 \%$ duty cycle response.

As a result, with the normalization suggested in this paper (Eq. (3)), expressing the decays in terms of $\mathrm{mV} / \mathrm{V}$ shows more similar starting and averaged magnitudes for the 50\% and 100\% IP decays (Fig. 2B). Despite the fact that the normalization proposed in Eq. (3) simplifies the comparison of the $50 \%$ and $100 \%$ signal levels, the shape of the IP curves differs significantly. In particular, the $100 \%$ IP decays defined through Eqs. (1), (2) and (3) are forced to small or negative data values at late times (Fig. 2), depending on the selected integration time for the $V_{D^{-}}$ value and the last IP gate respectively, even though the corresponding $50 \%$ IP responses have not reached their minimum. In this paper, this effect is accounted for by the modelling/inversion software, which includes the current waveform, the definition of the DC integration time and the full IP decays in the computations (Fiandaca et al. 2013, Fiandaca et al. 2012).

When looking at the comparison of non-normalized $100 \%$ and $50 \%$ duty cycle data a final consideration can be drawn about the signal-tonoise ratio. In fact, the noise in IP data depends on the nonnormalized signal level (Gazoty et al. 2013), and the almost doubled signal of the $100 \%$ duty cycle data can increase the signal-to-noise ratio by up to $100 \%$.

\section{Synthetic modelling}

Forward modelling and sensitivity analysis of resistivity and the Cole-Cole parameters for both the $50 \%$ and the $100 \%$ duty cycle waveforms were carried out on a 1D synthetic model (Fiandaca et al. 2013, Fiandaca et al. 2012). The sensitivity, $s$, is computed through the covariance of the estimator error for linear mapping described by Tarantola
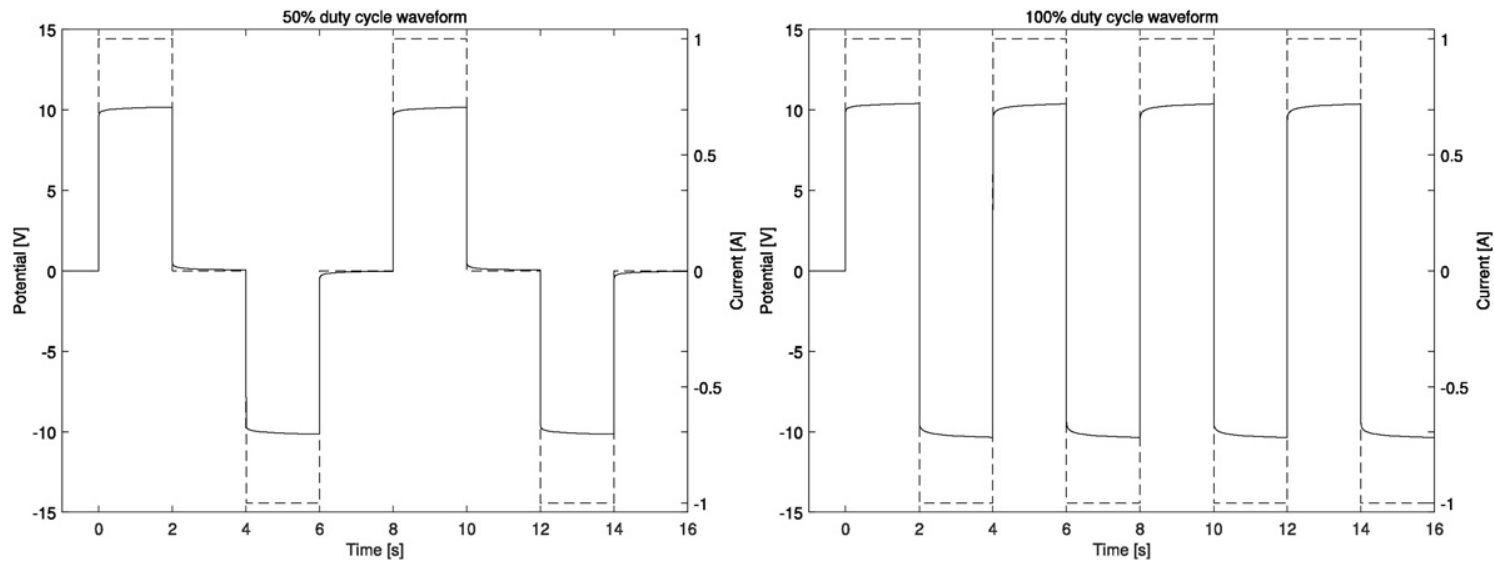

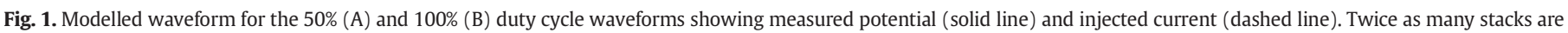
achieved with the $100 \%$ duty cycle compared with the $50 \%$ duty cycle with the same acquisition time. 

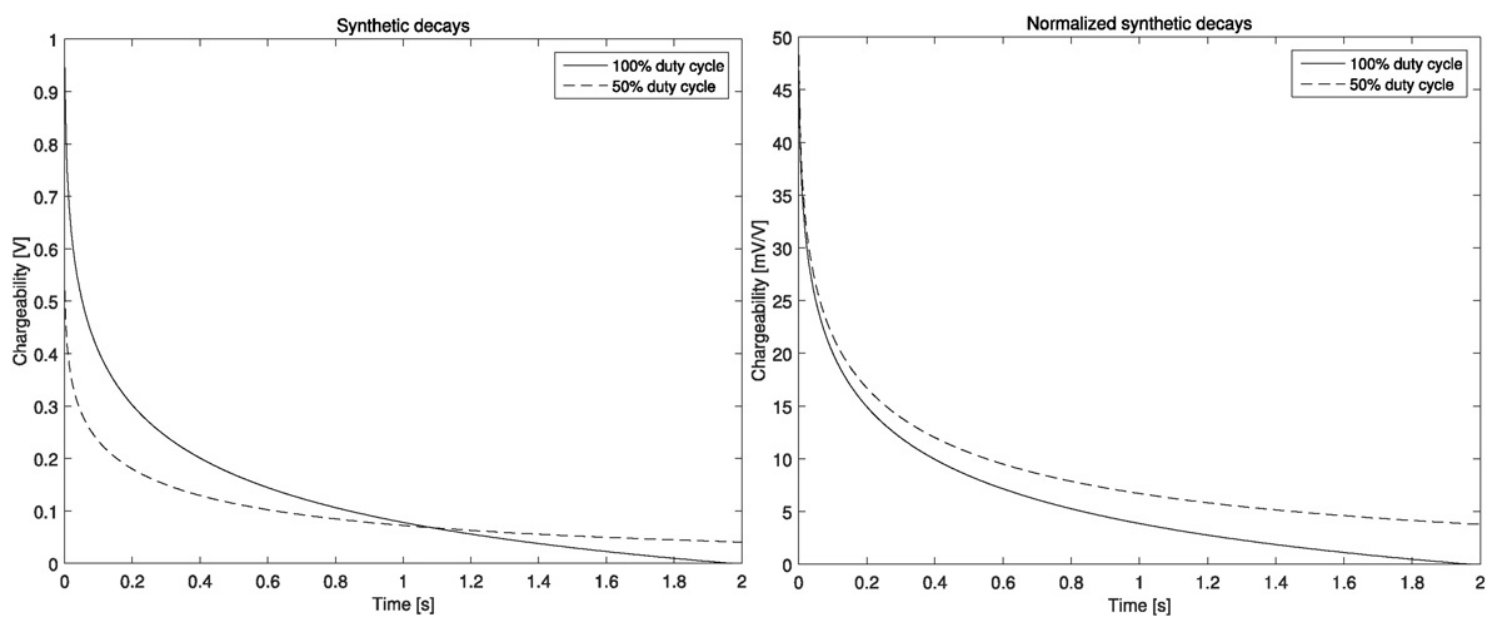

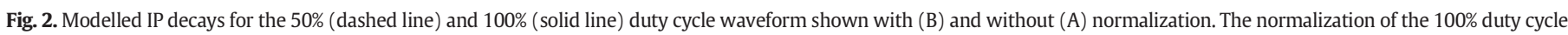

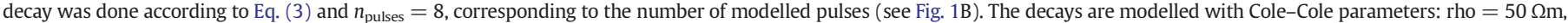
$m_{0}=100 \mathrm{mV} / \mathrm{V}, \tau=0.5 \mathrm{~s}$ and $C=0.3$.

and Valette (1982) and represents the relative uncertainty on the model parameters $p$ (because the analysis is performed on the logarithm of the model parameters):

$p / s \leq p \leq p \cdot s$

A synthetic three layer subsurface model (Table 1), with parameter ranges/contrasts similar to the ones found in the field example described in the next section, was used for the forward modelling of a Schlumberger vertical sounding with log-increasing electrode spacing and IP gate lengths. To emulate field conditions, a noise model identical to the one characterizing the field data was chosen for the sensitivity analysis. In particular, a relative standard deviation of $2 \%$ for the DC data and $10 \%$ for the IP data were used, together with a voltage dependent noise (Gazoty et al. 2013; Zhou and Dahlin 2003) that better describes the effect of the signal level on the data uncertainty. The voltage dependent noise model was controlled by a nominal voltage noise threshold $V_{\text {noise, }}$, defined for a nominal integration time Nominal $l_{\text {gatelength }}$ and stack size $n$ equal to one, and defined as:

$\sigma_{D C}=\frac{V_{\text {noise }}}{V_{D C}} \cdot \sqrt{\frac{\text { Nominal }_{\text {gatelength }}}{D C_{\text {gatelength }}} \cdot \frac{1}{n}}$

$\sigma_{I P}=\frac{V_{\text {noise }}}{V_{I P}} \cdot \sqrt{\frac{\text { Nominal }_{\text {gatelength }}}{I P_{\text {gatelength }}} \cdot \frac{1}{n}}$

A voltage threshold $V_{\text {noise }}=0.2 \mathrm{mV}$ with Nominal $_{\text {gatelength }}=0.01 \mathrm{~s}$ was used in the simulations.

The forward modelling and 1D sensitivity analysis was carried out by means of both of the waveforms and four different acquisition cases: two cases with the same on-time $\left(T_{\mathrm{on}}=4 \mathrm{~s}\right)$ and amount of stacks $(n=4)$ but with different waveforms (50\% and $100 \%$ duty cycles) and two other cases where either the on-time either the stacks were doubled for the $100 \%$ duty cycle waveform. In theory, the latter two cases give the same total duration of the acquisition as for the $50 \%$ duty cycle waveform (Table 2). Table 2 shows that the sensitivity for all

Table 1

Layer parameters used for the 1D subsurface model.

\begin{tabular}{lccccc}
\hline Layer & Thickness $(\mathrm{m})$ & $\rho(\Omega \mathrm{m})$ & $m_{0}(\mathrm{mV} / \mathrm{V})$ & $\tau(\mathrm{s})$ & $c(-)$ \\
\hline 1 & 8 & 250 & 40 & 1 & 0.3 \\
2 & 14 & 500 & 100 & 2 & 0.5 \\
3 & $\infty$ & 500 & 40 & 1 & 0.5
\end{tabular}

model parameters is comparable for all four acquisition cases, except for the relaxation time of layer two $(\tau-2)$ for which the first $(50 \%$ duty cycle, $T_{\mathrm{on}}=4 \mathrm{~s}, n=4$ ) and that the second last (100\% duty cycle, $T_{\text {on }}=8 \mathrm{~s}, n=4$ ) cases are more sensitive compared with the others.

\section{Field example}

The field data were acquired at a farmland with Quaternary deposits of clayey till overlaying a bedrock of Silurian shale. A known dolerite dike intruding the shale provided a chargeable IP structure for the experiment. The DCIP profile ( $157.5 \mathrm{~m}, 64$ electrodes with a spacing of $2.5 \mathrm{~m}$ ) was centred on top of the known IP anomaly and retrieved using a multiple gradient array protocol (Dahlin and Zhou 2006) with acid grade stainless steel electrodes and the Abem Terrameter LS instrument. This instrument continuously adapts the output voltage to keep a constant current with an accuracy of $0.4 \%$ (Abem 2011), and together with its capability of full-waveform acquisition is particularly well suited for this experiment. Separated cables were used for transmitting current and measuring potentials to reduce the effect of capacitive coupling and improve the IP data quality (Dahlin and Leroux 2012). The electrode contact resistance was measured for all electrodes with a mean value of $220 \Omega$ and a variation coefficient of approximately $45 \%$.

Both the $50 \%$ and the $100 \%$ duty cycle waveforms were used for DCIP measurements on the field setup. Except for the waveform duty cycle, all other settings were kept equal for the acquisitions: 1015 quadrupoles, $4 \mathrm{~s}$ on-time of current injection and 3 full stacks. This resulted in a total acquisition time of $154 \mathrm{~min}$ for the $50 \%$ duty cycle and 88 min for the $100 \%$ duty cycle, a reduction in acquisition time of approximately $43 \%$ for the $100 \%$ duty cycle waveform. The reason for the reduction not reaching the theoretical 50\% (only considering the duration of the waveforms) is that the instrument performs a number of test pulses prior to each current injection. These test pulses are part of the system that enables the instrument to maintain a constant current throughout the injection. In this study, these test pulses were not included in the full waveform modelling.

To get an accurate estimation of the IP response, the background potential variation during a current injection sequence needs to be accounted for. This is also essential for DC resistivity measurement and is commonly handled by measuring and averaging data from a positive-double negative-positive type duty cycle which acts as a filter that removes linear variation (Dahlin 2000), but it should be noted that a higher degree approach may be required in order to recover the more subtle spectral IP information. In this paper, a second order polynomial was adapted to the $\mathrm{V}_{\mathrm{DC}}$-values of the stacks and the non-constant terms 
Table 2

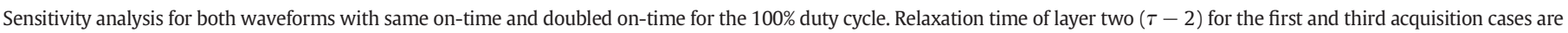
indicated with bold font.

\begin{tabular}{|c|c|c|c|c|c|c|c|c|c|c|c|c|c|c|}
\hline Duty cycle & $\begin{array}{l}T_{\text {on }} \\
(\mathrm{s})\end{array}$ & $\begin{array}{l}n \\
(-)\end{array}$ & $\begin{array}{l}\rho-1 \\
(\Omega \mathrm{m})\end{array}$ & $\begin{array}{l}\rho-2 \\
(\Omega \mathrm{m})\end{array}$ & $\begin{array}{l}\rho-3 \\
(\Omega \mathrm{m})\end{array}$ & $\begin{array}{l}m_{0}-1 \\
(\mathrm{mV} / \mathrm{V})\end{array}$ & $\begin{array}{l}m_{0}-2 \\
(\mathrm{mV} / \mathrm{V})\end{array}$ & $\begin{array}{l}m_{0}-3 \\
(\mathrm{mV} / \mathrm{V})\end{array}$ & $\begin{array}{l}\tau-1 \\
(\mathrm{~s})\end{array}$ & $\begin{array}{l}\tau-2 \\
(\mathrm{~s})\end{array}$ & $\begin{array}{l}\tau-3 \\
(\mathrm{~s})\end{array}$ & $\begin{array}{l}c-1 \\
(-)\end{array}$ & $\begin{array}{l}c-2 \\
(-)\end{array}$ & $\begin{array}{l}c-3 \\
(-)\end{array}$ \\
\hline $50 \%$ & 4 & 4 & 1.015 & 1.052 & 1.014 & 1.40 & 1.15 & 1.13 & 1.54 & 1.16 & 1.16 & 1.40 & 1.12 & 1.13 \\
\hline $100 \%$ & 4 & 4 & 1.015 & 1.052 & 1.014 & 1.47 & 1.17 & 1.16 & 1.54 & 1.27 & 1.17 & 1.47 & 1.14 & 1.16 \\
\hline $100 \%$ & 8 & 4 & 1.014 & 1.051 & 1.014 & 1.39 & 1.14 & 1.12 & 1.58 & 1.14 & 1.18 & 1.39 & 1.11 & 1.12 \\
\hline $100 \%$ & 4 & 8 & 1.015 & 1.052 & 1.014 & 1.47 & 1.17 & 1.16 & 1.54 & 1.27 & 1.16 & 1.47 & 1.14 & 1.15 \\
\hline
\end{tabular}

of this polynomial was used to correct the full waveform recording of measured potential.

Fig. 3 shows field data for both waveforms as apparent resistivity (Fig. 3A and C) and apparent chargeability (IP gate number 5, Fig. 3B and D) pseudosections. The resistivity data show comparable, in terms of spatial distribution and apparent resistivity values, pseudosections for both waveforms. The apparent chargeability pseudosections also have similar spatial distribution but the apparent chargeability section for the $100 \%$ duty cycle waveform shows slightly lower values than the $50 \%$ duty cycle waveform, when expressed as $\mathrm{mV} / \mathrm{V}$. The difference in magnitude of the apparent chargeability, which is also visible in the synthetic IP responses (Fig. 2B), can be explained by the different shapes of the $100 \%$ duty cycle waveform at late times, as discussed in Section 2.

Inversion of the field data shown in Fig. 3 were preformed for the spectral Cole-Cole parameters using the Aarhusinv code. The inversions were carried out with L1 model weights using the same inversion settings (noise model and vertical/horizontal weights) for both waveforms. This gave a final data misfit of 1.5 and 1.4 for the $50 \%$ and $100 \%$ duty cycle data respectively, when using a relative standard deviation of $2 \%$ for the DC data, $10 \%$ for the IP data and a voltage noise threshold of $0.2 \mathrm{mV}$.

Fig. 4 shows that the inversion models for the field data acquired with the two waveforms have comparable values for all model parameters and similar data misfit. The geometry of the parameter value distribution in the final inversion models is quite simple but the performance of the $50 \%$ and $100 \%$ duty cycle waveforms is expected to be comparable also when applied at environments with a more complex subsurface geometry. Similarly, are the performance of the waveforms expected to be comparable also in noiseier field conditions or possibly have better performance with the $100 \%$ duty cycle waveform due to higher signalto-noise ratio? (See Section 2).

Fig. 5 shows the data fit for the quadrupoles with lateral focus (i.e. horizontal position of median sensitivity) corresponding to the middle of the profile (Fig. 4). Similar to what is shown by the synthetic decays (Fig. 2), the acquired field data for the $100 \%$ duty cycle waveform are closer to zero for the later gates compared with the $50 \%$ duty cycle waveform.

The data acquired from the field tests (Figs. 3 and 5) generally show that the IP decays for the $50 \%$ and $100 \%$ duty cycle waveforms are different. As discussed above, this difference is in accordance with the synthetic modelling and depends on how the $100 \%$ duty cycle IP decay is defined. With an inversion software that includes the injected current waveform and the definitions for the $100 \%$ duty cycle IP decay, it is still possible to retrieve an inversion model from the $100 \%$ duty cycle data (Fig. 4B) that is similar to the inversion model from the $50 \%$ duty cycle waveform data (Fig. 4A).

\section{Conclusions}

Our results support that the approach of using a $100 \%$ duty cycle square waveform for current injection and measuring the IP response during the current on-time is practically applicable. We also show that the measuring time can be substantially reduced by the use of this method and that the signal-to-noise ratio will generally increase.

The results contribute to the usage of commercial IP measurements within areas where the time pressure on projects due to economic constraints is dominant. This is usually the case in engineering and environmental projects but decreasing the total measurement time and improving signal-to-noise ratio are beneficial for all DCIP surveys. Thus, an implementation of a commercial system which makes use of a $100 \%$ duty cycle and measures IP during the on-time could have several practical benefits. For example, it may encourage operators to make use of 3D-measurements which generally can be time demanding, to make use of more stacks in noisy environments to get better data quality or to increase the on-time at field sites where long time-constants are expected for the IP decays (such as for example landfills) to get additional spectral information.

The $100 \%$ duty cycle and on-time IP measurements described in this paper could be implemented as routine use in DCIP instruments and processing procedures. To actualize this, the instrument used need to
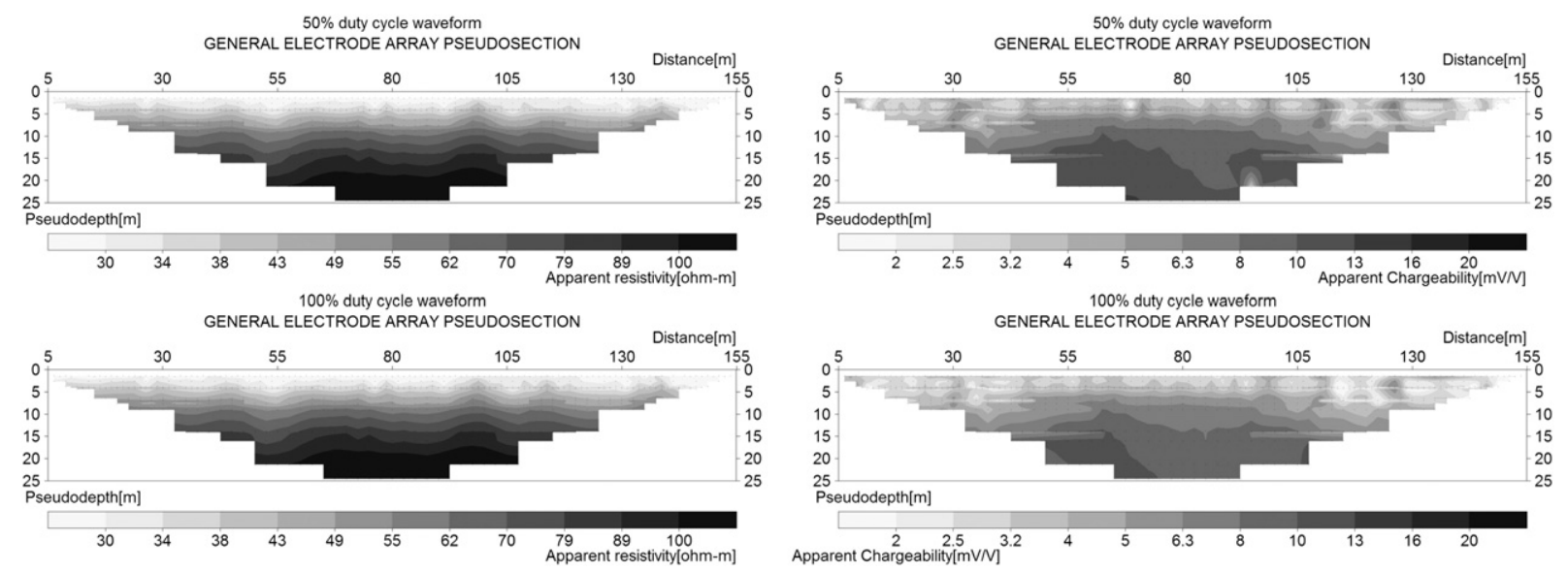

Fig. 3. DC pseudosections (A and C) and IP gate 5 (gate width of $143 \mathrm{~ms}$ and centre gate time of $376.5 \mathrm{~ms}$ ) for the $50 \%$ (B) and the $100 \%$ (D) duty cycle waveform. 

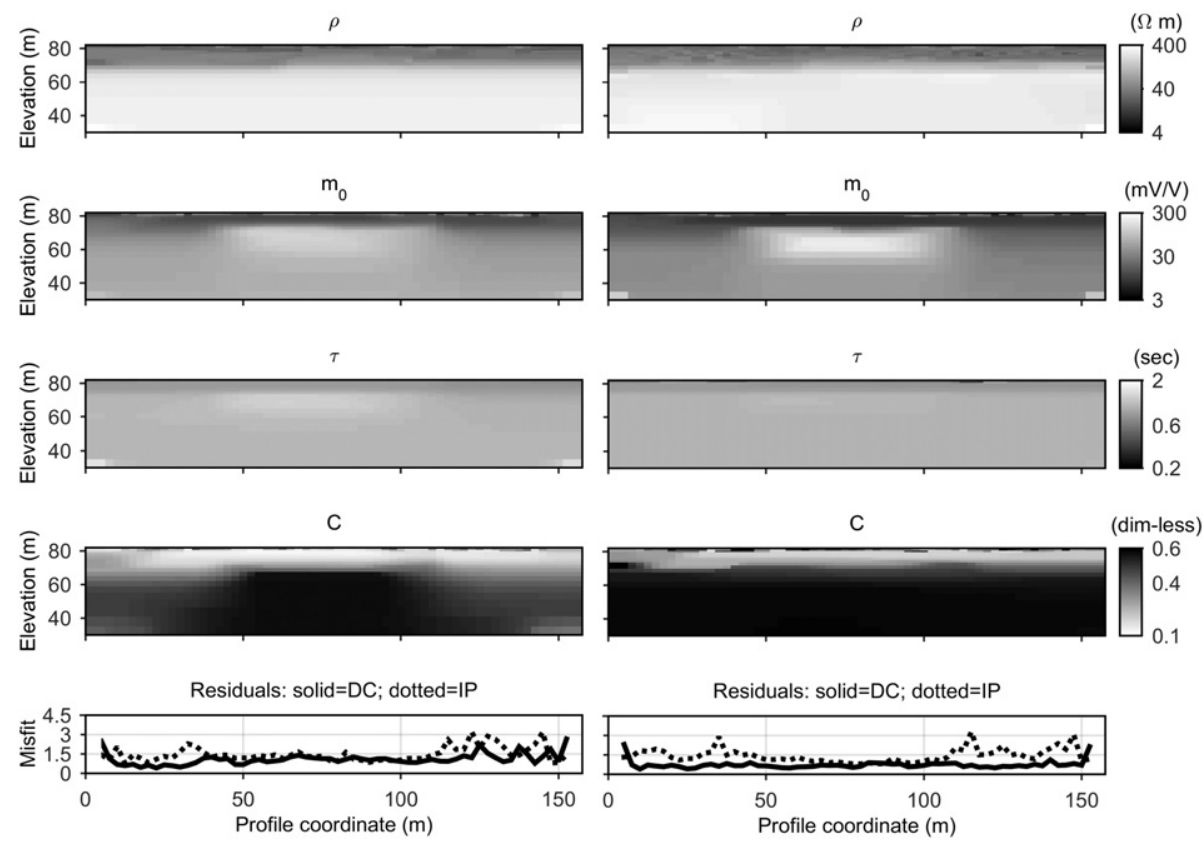

Fig. 4. Inverted sections and data misfit for field data acquired with the $50 \%$ (A) and $100 \%$ (B) duty cycle waveform.

be able to record full waveform information or be able to gate the data directly during the on-time. The instrument should also be able to keep a stable current waveform. If this is not possible, the waveform of the injected current needs to be fully described in the inversion software and included in the forward computations. These types of inversion software are also advantageous for comparing results from different waveforms ( $50 \%$ duty cycle versus $100 \%$ duty cycle).
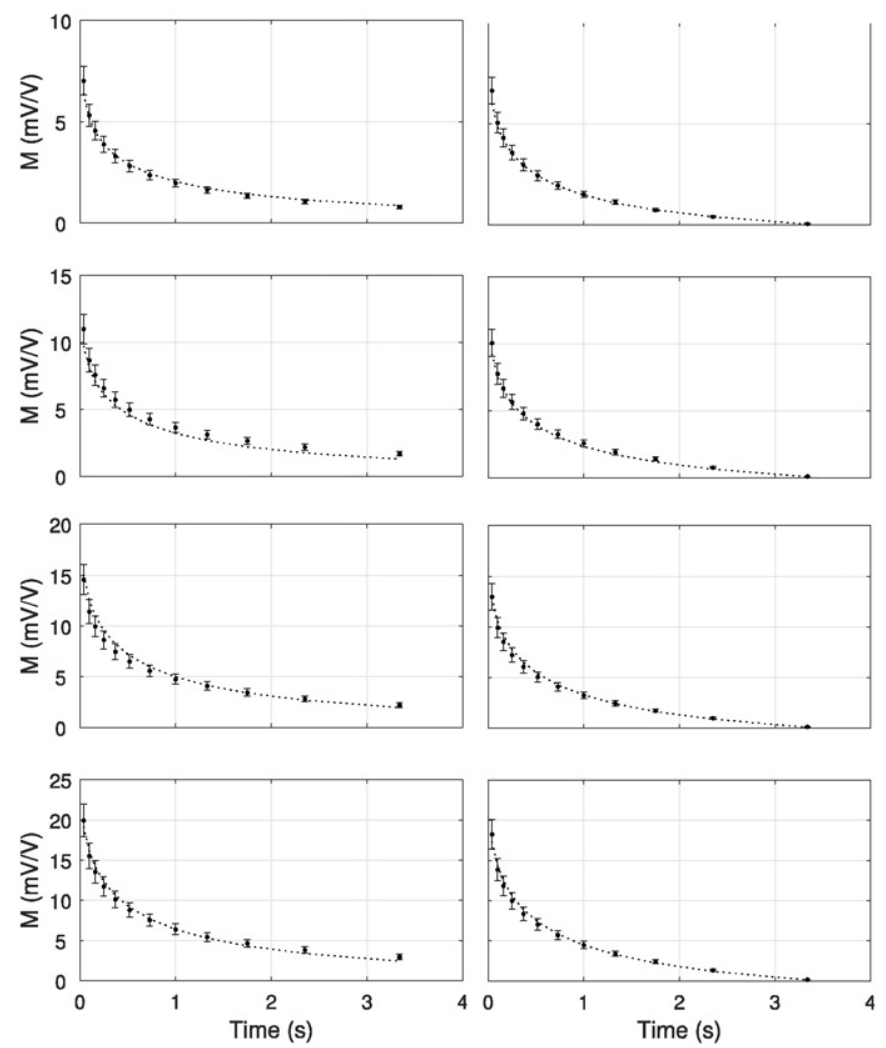

Fig. 5. IP data (points) with error bars (vertical lines) and corresponding forward response (dashed line) for the $50 \%$ (A) and 100\% (B) duty cycle waveforms. Data shown represents the middle of the field array with increasing pseudo depth from top to bottom.

\section{Acknowledgements}

Funding for the work was provided by Swedish Research Council Formas - The Swedish Research Council for Environment, Agricultural Sciences and Spatial Planning, (ref. 2012-1931), BeFo - Swedish Rock Engineering Research Foundation, (ref. 331) and SBUF - The Development Fund of the Swedish Construction Industry, (ref. 12719). The project is part of the Geoinfra-TRUST framework (http://www.trustgeoinfra.se/).

\section{References}

Abem, 2011. Terrameter LS Product Leaflet [www document]. URL http://www.abem. se/support/downloads/technical-specifications/terrameter-ls-leaflet-20111116 (accessed 5.27.14).

Auken, E., Christiansen, A.V., Kirkegaard, C., Fiandaca, G., Schamper, C., Behroozmand, A.A Binley, A., Nielsen, E., Effersø, F., Christensen, N.B., Sørensen, K., Foged, N., Vignoli, G. 2014. An overview of a highly versatile forward and stable inverse algorithm for airborne, ground-based and borehole electromagnetic and electric data. Explor. Geophys. http://dx.doi.org/10.1071/EG13097.

Dahlin, T., 2000. Short note on electrode charge-up effects in DC resistivity data acquisition using multi-electrode arrays. Geophys. Prospect. 48, 181-187. http://dx.doi. org/10.1046/j.1365-2478.2000.00172.x.

Dahlin, T., Leroux, V., 2010. Full wave form time-domain IP data acquisition, in: 16th European Meeting of Environmental and Engineering Geophysics. doi:http://dx.doi. org/10.3997/2214-4609.20144884

Dahlin, T., Leroux, V., 2012. Improvement in time-domain induced polarization data quality with multi-electrode systems by separating current and potential cables. Near Surf. Geophys. 10, 545-656. http://dx.doi.org/10.3997/1873-0604 2012028.

Dahlin, T., Zhou, B., 2006. Multiple-gradient array measurements for multichannel 2D resistivity imaging. Near Surf. Geophys. 4, 113-123. http://dx.doi.org/10.3997/18730604.2005037.

Doetsch, J., Fiandaca, G., Auken, E., Christiansen, A.V., Cahill, A.G., 2015a. Field-scale timedomain spectral induced polarization monitoring of geochemical changes induced by injected $\mathrm{CO}_{2}$ in a shallow aquifer. Geophysics 16, 10294. http://dx.doi.org/10.1190/ geo2014-0315.1.

Doetsch, J., Ingeman-Nielsen, T., Christiansen, A.V., Fiandaca, G., Auken, E., Elberling, B. 2015b. Direct current (DC) resistivity and induced polarization (IP) monitoring of active layer dynamics at high temporal resolution. Cold Reg. Sci. Technol. http://dx. doi.org/10.1016/j.coldregions.2015.07.002.

Fiandaca, G., Auken, E., Christiansen, A.V., Gazoty, A., 2012. Time-domain-induced polarization: full-decay forward modeling and 1D laterally constrained inversion of Cole-Cole parameters. Geophysics 77, E213-E225. http://dx.doi.org/10.1190/ geo2011-0217.1.

Fiandaca, G., Ramm, J., Binley, A., Gazoty, A., Christiansen, A.V., Auken, E., 2013. Resolving spectral information from time domain induced polarization data through 2-D inversion. Geophys. J. Int. 192, 631-646. http://dx.doi.org/10.1093/gji/ggs060. 
Gazoty, A., Fiandaca, G., Pedersen, J., Auken, E., Christiansen, A. V, 2012a. Mapping of landfills using time-domain spectral induced polarization data: the Eskelund case study. Near Surf. Geophys. 10, 575-586. doi:http://dx.doi.org/10.3997/1873-0604.2012046

Gazoty, A., Fiandaca, G., Pedersen, J., Auken, E., Christiansen, A. V, 2013. Data repeatability and acquisition techniques for time-domain spectral induced polarization. Near Surf. Geophys. 391-406. doi:http://dx.doi.org/10.3997/1873-0604.2013013

Gazoty, A., Fiandaca, G., Pedersen, J., Auken, E., Christiansen, A.V., Pedersen, J.K., 2012b. Application of time domain induced polarization to the mapping of lithotypes in a landfill site. Hydrol. Earth Syst. Sci. 16, 1793-1804. http://dx.doi.org/10.5194/hess16-1793-2012.

Slater, L.D., Lesmes, D., 2002. IP interpretation in environmental investigations. Geophysics 67, 77. http://dx.doi.org/10.1190/1.1451353.
Tarantola, A., Valette, B., 1982. Generalized nonlinear inverse problems solved using the least squares criterion. Rev. Geophys. http://dx.doi.org/10.1029/ RG020i002p00219.

Zhou, B., Dahlin, T., 2003. Properties and effects of measurement errors on 2D resistivity imaging surveying. Near Surf. Geophys. 1, 105-117. http://dx.doi.org/10.3997/18730604.2003001.

Zonge, K.L., Sauck, W.A., Sumner, J.S., 1972. Comparison of time, frequency, and phase measurements in induced polarization*. Geophys. Prospect. 20, 626-648. http://dx. doi.org/10.1111/j.1365-2478.1972.tb00658.x.

Zonge, K.L., Wynn, J., Urquhart, S., 2005. 9. Resistivity, induced polarization, and complex resistivity. Near-surface Geophysics, pp. 265-300 http://dx.doi.org/10. 1190/1.9781560801719.ch9. 